\title{
Image-based Synthesis for Deep 3D Human Pose Estimation
}

\author{
Grégory Rogez • Cordelia Schmid
}

Received: date / Accepted: date

\begin{abstract}
This paper addresses the problem of 3D human pose estimation in the wild. A significant challenge is the lack of training data, i.e., 2D images of humans annotated with 3D poses. Such data is necessary to train state-of-the-art CNN architectures. Here, we propose a solution to generate a large set of photorealistic synthetic images of humans with 3D pose annotations. We introduce an image-based synthesis engine that artificially augments a dataset of real images with 2D human pose annotations using 3D motion capture data. Given a candidate 3D pose, our algorithm selects for each joint an image whose 2D pose locally matches the projected $3 \mathrm{D}$ pose. The selected images are then combined to generate a new synthetic image by stitching local image patches in a kinematically constrained manner. The resulting images are used to train an end-to-end CNN for full-body 3D pose estimation. We cluster the training data into a large number of pose classes and tackle pose estimation as a $K$-way classification problem. Such an approach is viable only with large training sets such as ours. Our method outperforms most of the published works in terms of 3D pose estimation in controlled environments (Human3.6M) and shows promising results for real-world images (LSP). This demonstrates that CNNs trained on artificial images generalize well to real images. Compared to data generated from more classical rendering engines, our synthetic images do not require any domain adaptation or fine-tuning stage.
\end{abstract}

\section{Introduction}

Convolutional Neural Networks (CNN) have been very successful for many different tasks in computer vision. However,

Univ. Grenoble Alpes, Inria, CNRS, Grenoble INP*, LJK,

38000 Grenoble, France

* Institute of Engineering Univ. Grenoble Alpes

E-mail: gregory.rogez@inria.fr training these deep architectures requires large scale datasets which are not always available or easily collectable. This is particularly the case for 3D human pose estimation, for which an accurate annotation of 3D articulated poses in large collections of real images is non-trivial: annotating 2D images with 3D pose information is impractical [6] while large scale $3 \mathrm{D}$ pose capture is only available in constrained environments through marker-based (e.g., HumanEva [49], Human3.6M [20]) or makerless multiview systems (e.g., CMU Panoptic Dataset [25], MARCOnI Dataset [13]). The images captured in such conditions are limited in terms of subjects and environment diversity and do not match well real environments, i.e., real-world scenes with cluttered backgrounds. Moreover, with marker-based systems, the subjects have to wear capture suits with markers on them to which learning algorithms may overfit. This has limited the development of end-to-end CNN architectures for real-world 3D pose understanding.

Learning architectures usually augment existing training data by applying synthetic perturbations to the original images, e.g., jittering exemplars or applying more complex affine or perspective transformations [22]. Such data augmentation has proven to be a crucial stage, especially for training deep architectures. Recent work [21,37,54, 64] has introduced the use of data synthesis as a solution to train CNNs when only limited data is available. Synthesis can potentially provide infinite training data by rendering 3D CAD models from any camera viewpoint $[37,54,64]$. Fisher et al. [11] generate a synthetic "Flying Chairs" dataset to learn optical flow with a CNN and show that networks trained on this unrealistic data still generalize very well to existing datasets. In the context of scene text recognition, Jaderberg et al. [21] trained solely on data produced by a synthetic text generation engine. In this case, the synthetic data is highly realistic and sufficient to replace real data. Although synthesis seems like an appealing solution, there often exists a large 


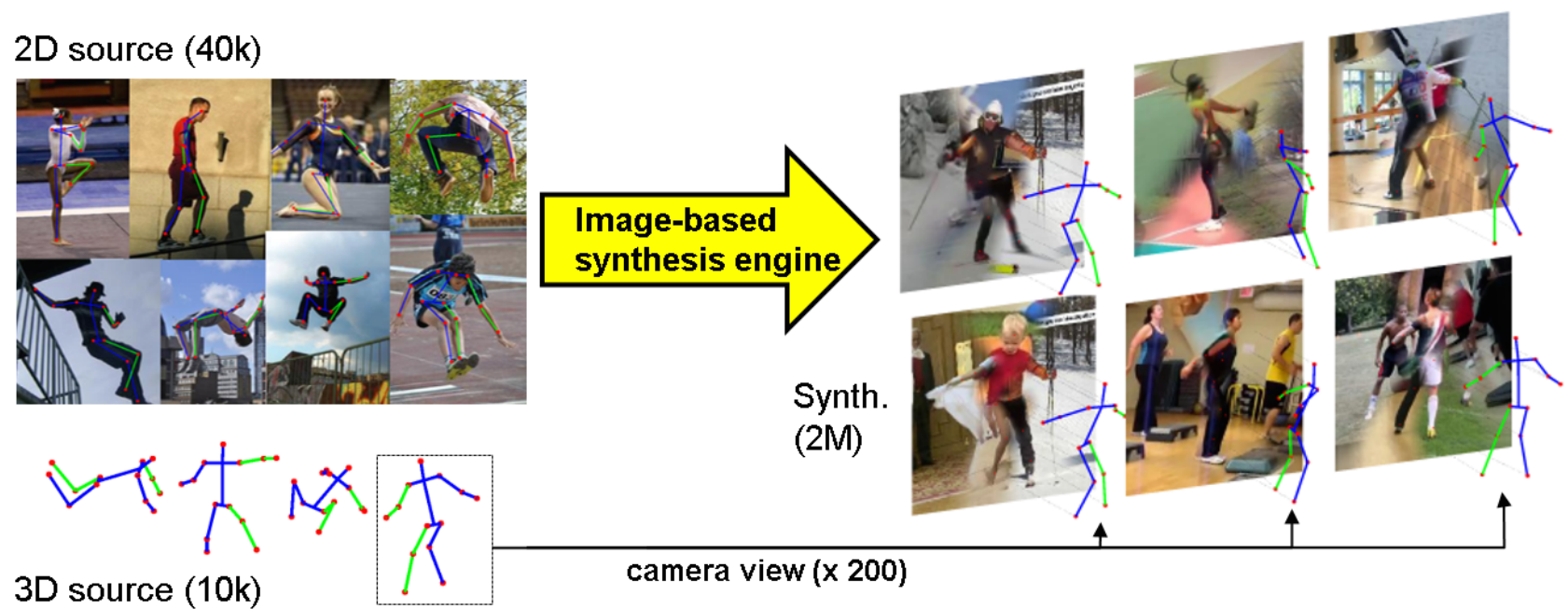

Fig. 1 Image-based synthesis engine. Input: real images with manual annotation of 2D poses, and 3D poses captured with a motion capture system. Output: 220x220 synthetic images and associated 3D poses.

domain shift from synthetic to real data [37]. Integrating a human 3D model in a given background in a realistic way is not trivial [20]. Rendering a collection of photo-realistic images (in terms of color, texture, context, shadow) that would cover the variations in pose, body shape, clothing and scenes is a challenging task.

Instead of rendering a human 3D model, we propose an image-based synthesis approach that makes use of motion capture data to augment an existing dataset of real images with $2 \mathrm{D}$ pose annotations. Our system synthesizes a very large number of new images showing more pose configurations and, importantly, it provides the corresponding 3D pose annotations (see Figure 1). For each candidate 3D pose in the motion capture library, our system combines several annotated images to generate a synthetic image of a human in this particular pose. This is achieved by "copy-pasting" the image information corresponding to each joint in a kinematically constrained manner. Given this large "in-the-wild" dataset, we implement an end-to-end CNN architecture for 3D pose estimation. Our approach first clusters the 3D poses into $K$ pose classes. Then, a $K$-way $\mathrm{CNN}$ classifier is trained to return a distribution over probable pose classes given a bounding box around the human in the image. Our method outperforms most state-of-the-art results in terms of 3D pose estimation in controlled environments and shows promising results on images captured "in-the-wild". The work presented in this paper is an extension of [42]. We provide an additional comparison of our image-based synthesis engine with a more classical approach based on rendering a human 3D model. The better performance of our method shows that for training a deep pipeline with a classification or a regression objective, it is more important to produce locally photorealistic data than globally coherent data.

\subsection{Related work}

3D human pose estimation in monocular images. Recent approaches employ CNNs for 3D pose estimation in monocular images [8,28,36] or in videos [71]. Due to the lack of large scale training data, they are usually trained (and tested) on 3D motion capture data in constrained environments [28]. Pose understanding in natural images is usually limited to $2 \mathrm{D}$ pose estimation $[9,59,60]$. Motivated by these well-working off-the-shelf 2D detectors and inspired by earlier work in single view 3D pose reconstruction [33,40,50,55], recent work also tackles $3 \mathrm{D}$ pose understanding from $2 \mathrm{D}$ poses $[2$, $7,15,32,58]$. Some approaches use as input the $2 \mathrm{D}$ joints automatically provided by a 2D pose detector [7,32, 52, 62], while others jointly solve the $2 \mathrm{D}$ and $3 \mathrm{D}$ pose estimation [51, 58,68]. Most similar to ours are the architectures that take advantage of the different sources of training data, i.e., indoor images with motion capture 3D poses and real-world images with 2D annotations [31,67,69]. Iqbal et al. [67] use a dual-source approach that combines $2 \mathrm{D}$ pose estimation with 3D pose retrieval. Mehta et al. [31] propose a 2D-to-3D knowledge transfer to generalize to in-the-wild images, using pre-trained $2 \mathrm{D}$ pose networks to initialize the $3 \mathrm{D}$ pose regression networks. The architecture of [69] shares the common representations between the $2 \mathrm{D}$ and the 3D tasks. Our method uses the same two training sources, i.e., images with annotated 2D pose and 3D motion capture data. However, we combine both sources off-line to generate a large training set that is used to train an end-to-end CNN 3D pose classifier. This is shown to improve over [67], which can be explained by the fact that training is performed in an end-to-end fashion. Synthetic pose data. A number of works have considered the use of synthetic data for human pose estimation. Synthetic data have been used for upper body [47], full-body silhouettes [1], hand-object interactions [45], full-body pose 


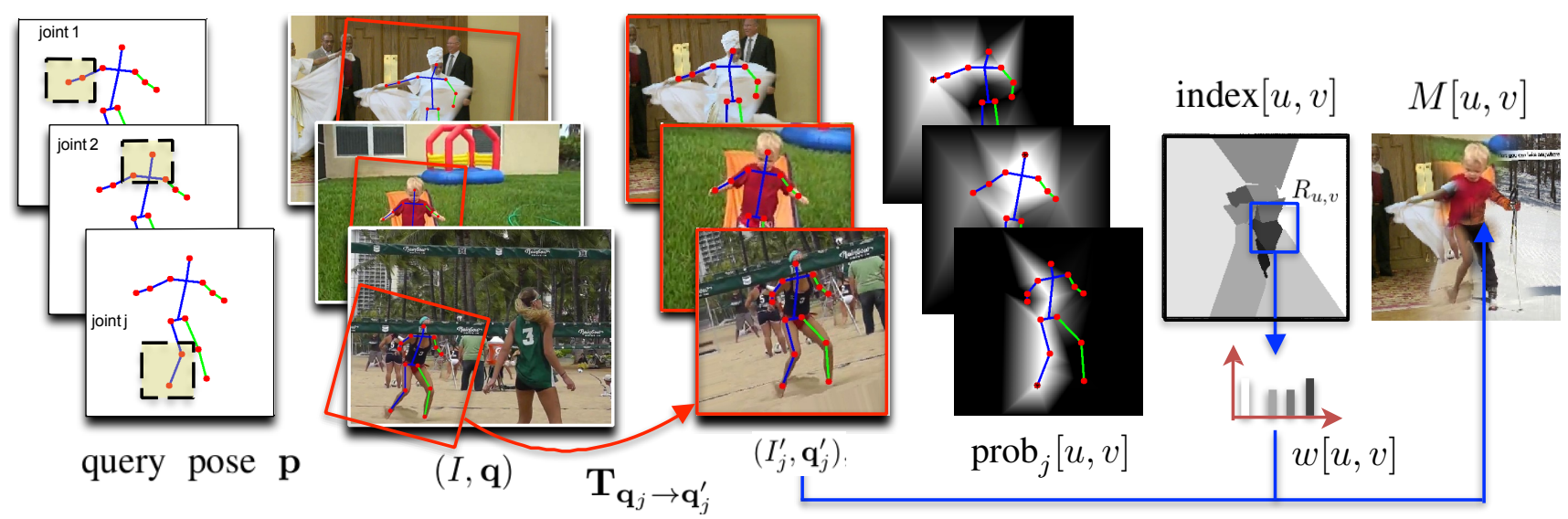

Fig. 2 Synthesis engine. From left to right: for each joint $j$ of a $2 \mathrm{D}$ query pose $\mathbf{p}$ (centered in a $220 \times 220$ bounding box), we align all the annotated 2D poses w.r.t the limb and search for the best pose match, resulting in a list of $n$ matches $\left\{\left(I_{j}^{\prime}, \mathbf{q}_{j}^{\prime}\right), j=1 \ldots n\right\}$ where $I_{j}^{\prime}$ is obtained after transforming $I_{j}$ with $\mathbf{T} \mathbf{q}_{j} \rightarrow \mathbf{q}_{j}^{\prime}$. For each retrieved pair, we compute a probability map prob $j[u, v]$. These $n$ maps are used to compute index $[u, v] \in\{1 \ldots n\}$, pointing to the image $I_{j}^{\prime}$ that should be used for a particular pixel $(u, v)$. Finally, our blending algorithm computes each pixel value of the synthetic image $M[u, v]$ as the weighted sum over all aligned images $I_{j}^{\prime}$, the weights being calculated using a histogram of indexes in a squared region $R_{u, v}$ around $(u, v)$.

from depth [48] or egocentric RGB-D scenes [43]. Zuffi and Black [72] used a 3D mesh-model to sample synthetic exemplars and fit 3D scans. Recently, Chen et al. [8] trained a human 3D pose regressor on synthetic training images rendered from such a 3D mesh-model. Similarly, [19] trained a human detector for unusual pedestrian using synthetic data generated by a game engine. In both cases, a domain adaptation stage was necessary to generalize to real images. In [17], a scene-specific pedestrian detector was learned without real data while [14] synthesized virtual samples with a generative model to enhance the classification performance of a discriminative model. In [18], pictures of 2D characters were animated by fitting and deforming a 3D mesh model. Later, [39] augmented labelled training images with small perturbations in a similar way. These methods require a perfect segmentation of the humans in the images. Park and Ramanan [35] synthesized hypothetical poses for tracking by applying geometric transformations to the first frame of a video sequence. We also use image-based synthesis to generate images but our rendering engine combines image regions from several images to create images with associated $3 \mathrm{D}$ poses.

\section{Image-based synthesis engine}

At the heart of our approach is an image-based synthesis engine that artificially generates "in-the-wild" images with 3D pose annotations. Our method takes as input a dataset of real images with $2 \mathrm{D}$ annotations and a library of 3D motion capture data, and generates a large number of synthetic images with associated 3D poses (see Figure 1). We introduce an image-based rendering engine that augments the existing database of annotated images with a very large set of pho- torealistic images covering more body pose configurations than the original set. This is done by projecting the motion capture 3D poses on random camera views to obtain a set of 2D poses for which new images are synthesized by selecting and stitching image patches in a kinematically constrained manner. Our synthesis process consists of two stages: the mosaic construction stage that selects and stitches image patches together and a pose-aware blending process that improves image quality and erases patch seams. These are discussed in the following subsections. Figure 2 summarizes our synthesis process.

\subsection{Motion capture guided image mosaicing}

Given a 3D pose with $n$ joints $\mathbf{P} \in \mathbb{R}^{n \times 3}$, and its projected $2 \mathrm{D}$ joints $\mathbf{p}=\left\{p_{j}, j=1 \ldots n\right\}$ in a particular camera view, we want to find for each joint $j \in\{1 \ldots n\}$ an image whose annotated 2D pose presents a similar kinematic configuration around $j$. To do so, we define a distance function between 2 different $2 \mathrm{D}$ poses $\mathbf{p}$ and $\mathbf{q}$, conditioned on joint $j$ as:

$D_{j}(\mathbf{p}, \mathbf{q})=\sum_{k=1}^{n} d_{\mathrm{E}}\left(p_{k}, q_{k}^{\prime}\right)$

where $d_{\mathrm{E}}$ is the Euclidean distance. $\mathbf{q}^{\prime}$ is the aligned version of $\mathbf{q}$ with respect to joint $j$ after applying a rigid transformation $\mathbf{T}_{\mathbf{q}_{j} \rightarrow \mathbf{q}_{j}^{\prime}}$, which respects $q_{j}^{\prime}=p_{j}$ and $q_{i}^{\prime}=p_{i}$, where $i$ is the farthest directly connected joint to $j$ in $\mathbf{p}$. The rigid transformation $\mathbf{T}_{\mathbf{q}_{j} \rightarrow \mathbf{q}_{j}^{\prime}}$ is obtained by combining the translation $\mathbf{t}_{q_{j} \rightarrow p_{j}}$ aligning joint $j$ in $\mathbf{p}$ and $\mathbf{q}$, and the rotation matrix $\mathbf{R}_{q_{i} \rightarrow p_{i}}$ aligning joint $i$ in $\mathbf{p}$ and $\mathbf{q}$ :

$\mathbf{T}_{\mathbf{q}_{j} \rightarrow \mathbf{q}_{j}^{\prime}}\left(p_{k}\right)=\mathbf{R}_{q_{i} \rightarrow p_{i}}\left(p_{k}\right)+\mathbf{t}_{q_{j} \rightarrow p_{j}}$. 
An example of such transformation is given in Fig. 3.

The function $D_{j}$ measures the similarity between 2 joints by aligning and taking into account the entire poses. To increase the influence of neighboring joints, we weight the distances $d_{\mathrm{E}}$ between each pair of joints $\left\{\left(p_{k}, q_{k}^{\prime}\right), k=1 \ldots n\right\}$ according to their distance to the query joint $j$ in both poses. Eq. 1 becomes:

$D_{j}(\mathbf{p}, \mathbf{q})=\sum_{k=1}^{n}\left(w_{k}^{j}(\mathbf{p})+w_{k}^{j}(\mathbf{q})\right) d_{\mathrm{E}}\left(p_{k}, q_{k}^{\prime}\right)$

where weight $w_{k}^{j}$ is inversely proportional to the distance between joint $k$ and the query joint $j$, i.e., $w_{k}^{j}(\mathbf{p})=1 / d_{\mathrm{E}}\left(p_{k}, p_{j}\right)$ and normalized so that $\sum_{k} w_{k}^{j}(\mathbf{p})=1$. This cost function is illustrated in Fig. 3.

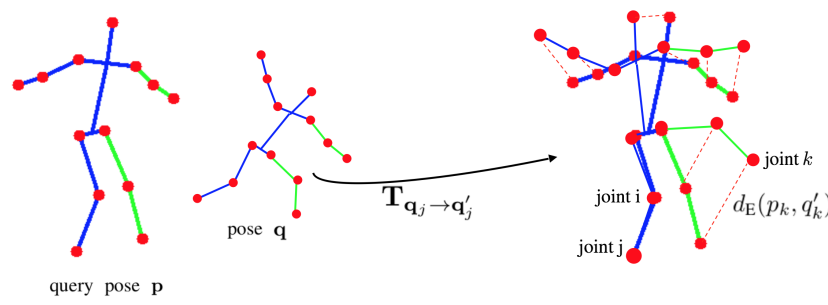

Fig. 3 Illustration of the cost function employed to find pose matches. We show two poses aligned at joint $j$ with red lines across all the other joints denoting contributors to the distance.

For each joint $j$ of the query pose $\mathbf{p}$, we retrieve from our dataset $\mathbb{Q}=\left\{\left(I_{1}, \mathbf{q}_{1}\right) \ldots\left(I_{N}, \mathbf{q}_{N}\right)\right\}$ of images and annotated 2D poses:

$\mathbf{q}_{j}=\operatorname{argmin}_{\mathbf{q} \in \mathbb{Q}} D_{j}(\mathbf{p}, \mathbf{q}) \quad \forall j \in\{1 \ldots n\}$.

In practice, we do not search for self-occluded joints, i.e., joints occluded by another body part, that can be labelled as such by simple 3D reasoning. We obtain a list of $n$ matches $\left\{\left(I_{j}^{\prime}, \mathbf{q}_{j}^{\prime}\right), j=1 \ldots n\right\}$ where $I_{j}^{\prime}$ is the cropped image obtained after transforming $I_{j}$ with $\mathbf{T}_{\mathbf{q}_{j} \rightarrow \mathbf{q}_{j}^{\prime}}$. Note that a same pair $(I, \mathbf{q})$ can appear multiple times in the list of candidates, i.e., being a good match for several joints.

Finally, to render a new image, we need to select the candidate images $I_{j}^{\prime}$ to be used for each pixel $(u, v)$. Instead of using regular patches, we compute a probability map $p_{j}[u, v]$ associated with each pair $\left(I_{j}^{\prime}, \mathbf{q}_{j}^{\prime}\right)$ based on local matches measured by $d_{\mathrm{E}}\left(p_{k}, q_{k}^{\prime}\right)$ in Eq. 1 . To do so, we first apply a Delaunay triangulation to the set of $2 \mathrm{D}$ joints in $\left\{\mathbf{q}_{j}^{\prime}\right\}$ obtaining a partition of the image into triangles, according to the selected pose. Then, we assign the probability $\operatorname{prob}_{j}\left(q_{k}^{\prime}\right)=\exp \left(-d_{\mathrm{E}}\left(p_{k}, q_{k}^{\prime}\right)^{2} / \sigma^{2}\right)$ to each vertex $q_{k}^{\prime}$. We finally compute a probability map $\operatorname{prob}_{j}[u, v]$ by interpolating values from these vertices using barycentric interpolation inside each triangle. The resulting $n$ probability maps are concatenated and an index map index $[u, v] \in\{1 \ldots n\}$ can be computed as follows:

$\operatorname{index}[u, v]=\operatorname{argmax}_{j \in\{1 \ldots n\}} \operatorname{prob}_{j}[u, v]$,

this map pointing to the training image $I_{j}^{\prime}$ that should be used for each pixel $(u, v)$. A mosaic $M[u, v]$ can be generated by "copy-pasting" image information at pixel $(u, v)$ indicated by index $[u, v]$ :

$M[u, v]=I_{j^{*}}^{\prime}[u, v] \quad$ with $\quad j^{*}=\operatorname{index}[u, v]$.

\subsection{Pose-aware image blending}

The mosaic $M[u, v]$ resulting from the previous stage presents significant artifacts at the boundaries between image regions. Smoothing is necessary to prevent the learning algorithm from interpreting these artifacts as discriminative pose-related features. We first experimented with off-theshelf image filtering and alpha blending algorithms, but the results were not satisfactory. Instead, we propose a new poseaware blending algorithm that maintains image information on the human body while erasing most of the stitching artifacts. For each pixel $(u, v)$, we select a surrounding squared region $R_{u, v}$ whose size varies with the distance $d_{u, v}$ of pixel $(u, v)$ to the pose:

$R_{u, v}=\alpha+\beta d_{u, v}$.

$R_{u, v}$ will be larger when far from the body and smaller nearby. The distance $d_{u, v}$ is computed using a distance transform to the rasterisation of the $2 \mathrm{D}$ skeleton. In this paper, we empirically set $\alpha=6$ pixels and $\beta=0.25$ to synthesise $220 \times 220$ images. Then, we evaluate how much each image $I_{j}^{\prime}$ should contribute to the value of pixel $(u, v)$ by building a histogram of the image indexes inside the region $R_{u, v}$ :

$w[u, v]=\operatorname{Hist}\left(\operatorname{index}\left(R_{u, v}\right)\right)$,

where the weights are normalized so that $\sum_{j} w_{j}[u, v]=1$. The final mosaic $M[u, v]$ (see examples in Figure 1) is then computed as the weighted sum over all aligned images:

$M[u, v]=\sum_{j} w_{j}[u, v] I_{j}^{\prime}[u, v]$.

This procedure produces plausible images that are kinematically correct and locally photorealistic. See examples presented in Figure 1 and Figure 4. 

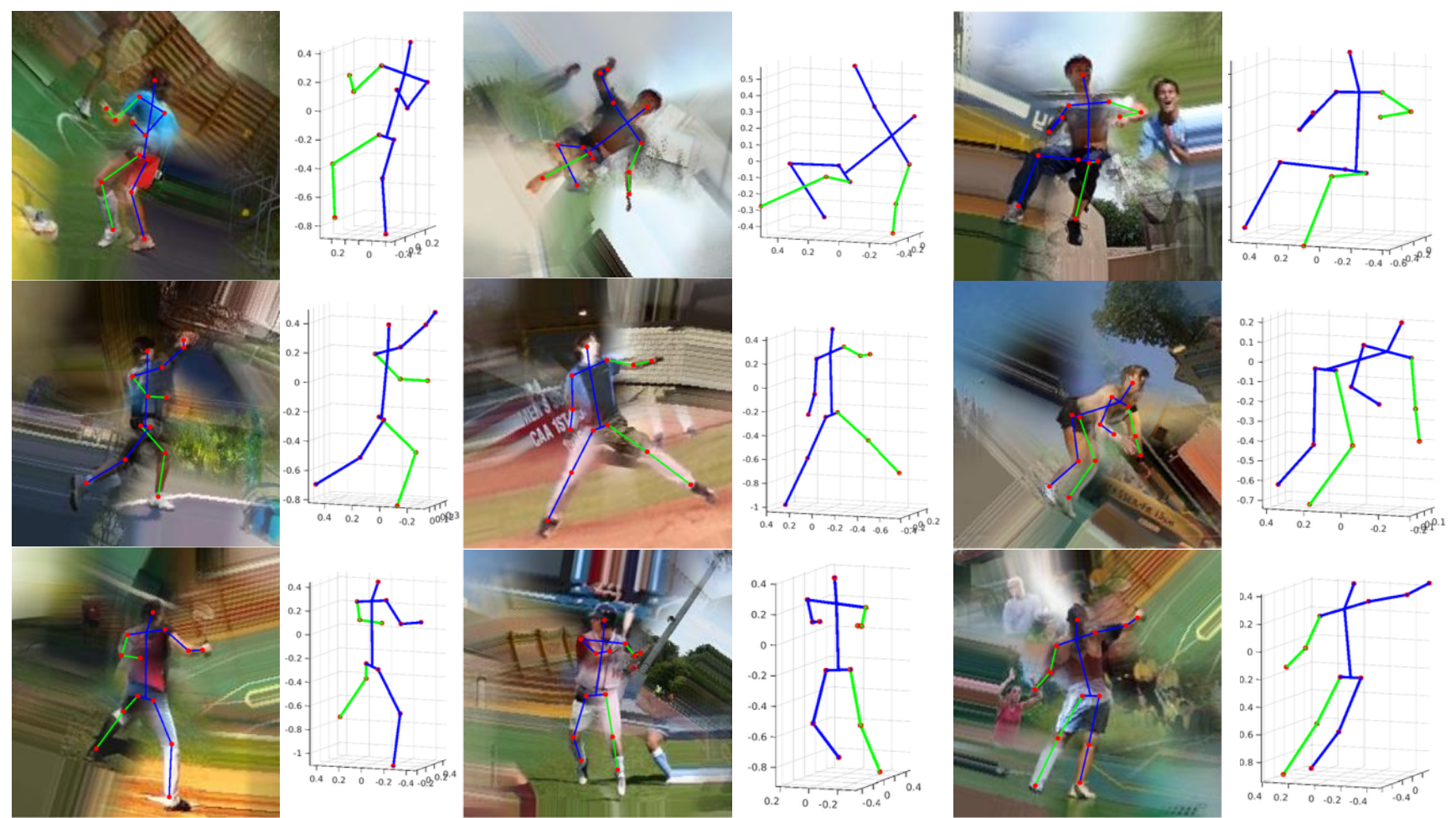

Fig. 4 Examples of synthetics images generated using Leeds Sport dataset (LSP)[23] and CMU motion capture dataset as 2D and 3D sources respectively. For each case, we show the $2 \mathrm{D}$ pose overlaid on the image and the corresponding orientated 3D pose.

\section{CNN for full-body $3 D$ pose estimation}

Human pose estimation has been addressed as a classification problem in the past $[4,34,41,43]$. Here, the 3D pose space is partitioned into $K$ clusters and a $K$-way classifier is trained to return a distribution over pose classes. Such a classification approach allows modeling multimodal outputs in ambiguous cases, and produces multiple hypothesis that can be rescored, e.g., using temporal information. Training such a classifier requires a reasonable amount of data per class which implies a well-defined and limited pose space (e.g. walking action) [4,41], a large-scale synthetic dataset [43] or both [34]. Here, we introduce a CNN-based classification approach for full-body 3D pose estimation. Inspired by the DeepPose algorithm [60] where the AlexNet CNN architecture [27] is used for full-body 2D pose regression, we select the same architecture and adapt it to the task of 3D body pose classification. This is done by adapting the last fullyconnected layer to output a distribution of scores over pose classes as illustrated in Figure 5. Training such a classifier requires a large amount of training data that we generate using our image-based synthesis engine.

Given a library of motion capture data and a set of camera views, we synthesize for each $3 \mathrm{D}$ pose a $220 \times 220$ image. This size has proved to be adequate for full-body pose estimation [60]. The 3D poses are then aligned with respect to the camera center and translated to the center of the torso, i.e., the average position between shoulders and hips coordinates. In that way, we obtain orientated $3 \mathrm{D}$ poses that also contain the viewpoint information. We cluster the resulting $3 \mathrm{D}$ poses to define our classes which will correspond to groups of similar orientated 3D poses, i.e., body pose configuration and camera viewpoint. We empirically found that $K=5000$ clusters was a sufficient number of clusters and that adding more clusters did not further improve the results. For evaluation, we return the average $2 \mathrm{D}$ and $3 \mathrm{D}$ poses of the top scoring class.

To compare with [60], we also train a holistic pose regressor, which regresses to $2 \mathrm{D}$ and $3 \mathrm{D}$ poses (not only $2 \mathrm{D}$ ). To do so, we concatenate the $3 \mathrm{D}$ coordinates expressed in meters normalized to the range $[-1,1]$, with the $2 \mathrm{D}$ pose coordinates, also normalized in the range $[-1,1]$ following [60].

\section{Experiments}

We address 3D pose estimation in the wild. However, there does not exist a dataset of real-world images with 3D annotations. We thus evaluate our method in two different settings using existing datasets: (1) we validate our 3D pose predictions using Human3.6M [20] which provides accurate 3D and $2 \mathrm{D}$ poses for 15 different actions captured in a controlled indoor environment; (2) we evaluate on the Leeds Sport dataset (LSP) [23] that presents real-world images together with full-body $2 \mathrm{D}$ pose annotations. We demonstrate competitive results with state-of-the-art methods for both of them. 


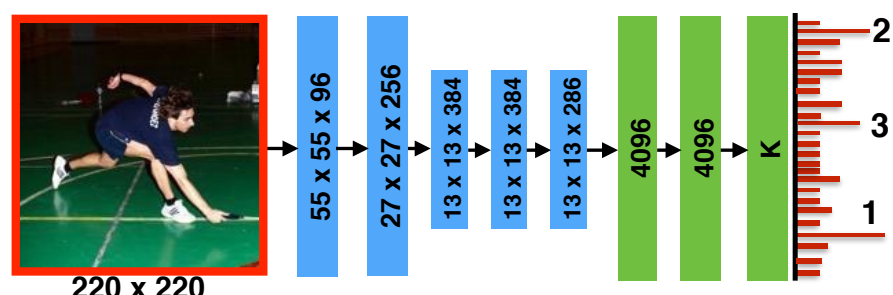

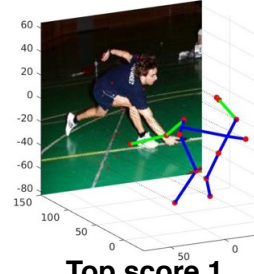

Top score 1

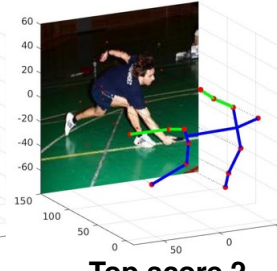

Top score 2

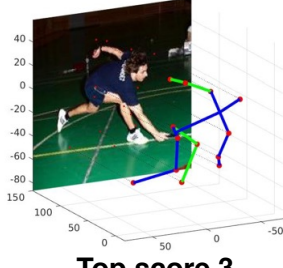

Top score 3

Fig. 5 CNN-based pose classifier. We show the different layers and their corresponding dimensions, with convolutional layers depicted in blue and fully connected ones in green. The output is a distribution over $\mathrm{K}$ pose classes. Pose estimation is obtained by taking the highest score in this distribution. We show on the right the 3D poses for 3 highest scores. For this example, the top scoring class (top score 1) is correct.

Table 1 Impact of synthetic data on the performances for the regressor and the classifier. The 3D pose estimation results are given following the protocol P1 of Human3.6M (see text for details).

\begin{tabular}{l|ccccc}
\hline Method & $\begin{array}{c}\text { Type } \\
\text { of images }\end{array}$ & $\begin{array}{c}\text { 2D source } \\
\text { size }\end{array}$ & $\begin{array}{c}\text { 3D source } \\
\text { size }\end{array}$ & $\begin{array}{c}\text { Training } \\
\text { pairs }\end{array}$ & $\begin{array}{c}\text { Error } \\
(\mathrm{mm})\end{array}$ \\
\hline Reg. & Real & 17,000 & 17,000 & 17,000 & 112.9 \\
Class. & Real & 17,000 & 17,000 & 17,000 & 149.7 \\
\hline Reg. & Synth & 17,000 & 190,000 & 190,000 & 101.9 \\
Class. & Synth & 17,000 & 190,000 & 190,000 & 97.2 \\
\hline Reg. & Real & 190,000 & 190,000 & 190,000 & 139.6 \\
Class. & Real & 190,000 & 190,000 & 190,000 & 97.7 \\
\hline Reg. & Synth + Real & 207,000 & 190,000 & 380,000 & 125.5 \\
Class. & Synth + Real & 207,000 & 190,000 & 380,000 & $\mathbf{8 8 . 1}$ \\
\hline
\end{tabular}

Our image-based rendering engine requires two different training sources: 1) a 2D source of images with 2D pose annotations and 2) a motion capture 3D source. We consider two different datasets for each: for 3D poses we use the CMU motion capture dataset ${ }^{1}$ and the Human3.6M 3D poses [20], and for 2D pose annotations the MPII-LSP-extended dataset [38] and the Human3.6M 2D poses and images.

Motion capture 3D source. The CMU motion capture dataset consists of 2500 sequences and a total of 140,000 3D poses. We align the $3 \mathrm{D}$ poses w.r.t. the torso and select a subset of 12,000 poses, ensuring that selected poses have at least one joint $5 \mathrm{~cm}$ apart. In that way, we densely populate our pose space and avoid repeating common poses, e.g., neutral standing or walking poses which are over-represented in the dataset. For each of the 12,000 original motion capture poses, we sample 180 random virtual views with azimuth angle spanning 360 degrees and elevation angles in the range $[-45,45]$. We generate over 2 million pairs of 3D/2D pose configurations (articulated poses + camera position and angle). For Human3.6M, we randomly selected a subset of 190,000 orientated 3D poses, discarding similar poses, i.e., when the average Euclidean distance of the joints is less than $15 \mathrm{~mm}$ as in [67].

2D source. For the training dataset of real images with $2 \mathrm{D}$ pose annotations, we use the MPII-LSP-extended [38] which is a concatenation of the extended LSP [24] and the MPII dataset [3]. Some of the poses were manually corrected as a non-negligible number of annotations are not accurate enough or completely wrong (eg., right-left inversions or

\footnotetext{
${ }^{1}$ http://mocap.cs.cmu.edu
}

bad ordering of the joints along a limb). We mirror the images to double the size of the training set, obtaining a total of 80,000 images with 2D pose annotations. For Human3.6M, we consider the 4 cameras and create a pool of 17,000 images and associated $2 \mathrm{D}$ poses that we also mirror. To create a diverse set of images, we ensure that the maximum joint-tojoint distance between corresponding 3D poses is over $5 \mathrm{~cm}$, i.e., similar poses have at least one joint $5 \mathrm{~cm}$ apart in $3 \mathrm{D}$.

\subsection{Evaluation on Human3.6M Dataset}

To compare our results with recent work in 3D pose estimation [67], we follow the protocol introduced in [26] and employed in [67]: we consider six subjects (S1, S5, S6, S7, S8 and S9) for training, use every $64^{\text {th }}$ frame of subject S11 for testing and evaluate the 3D pose error ( $\mathrm{mm}$ ) averaged over the 13 joints. We refer to this protocol by P1. As in [67], we measure a $3 \mathrm{D}$ pose error that aligns the pose by a rigid transformation, but we also report the absolute error.

We first evaluate the impact of our synthetic data on the performances for both the regressor and classifier. The results are reported in Table 1. We can observe that when considering few training images $(17,000)$, the regressor clearly outperforms the classifier which, in turns, reaches better performances when trained on larger sets. This can be explained by the fact that the classification approach requires a sufficient amount of examples. We, then, compare results when training both regressor and classifier on the same 190,000 poses considering a) synthetic data generated from Human3.6M, b) the real images corresponding to the 190,000 poses and 

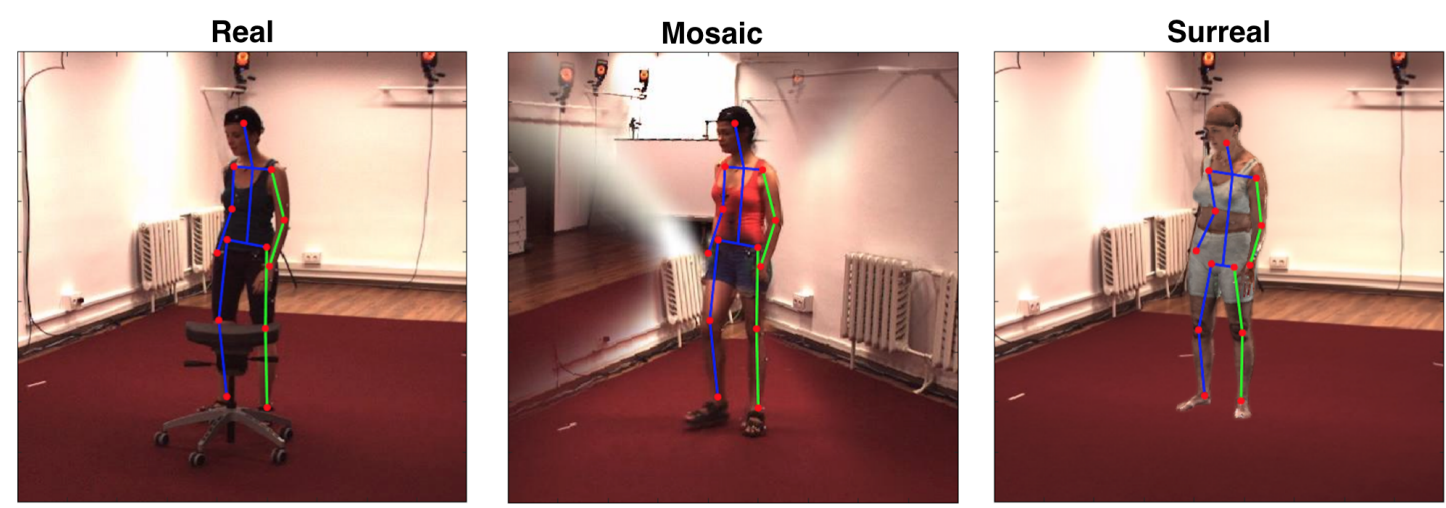

Fig. 6 Human 3.6M real and synthetic data. We show on the left a training image from protocol 2 with the overlayed $2 \mathrm{D}$ pose. In the middle, we show a "mosaic" image synthetized using the $2 \mathrm{D}$ pose from the real image on the left. In this case, the mosaic has been built by stitching image patches from 3 different subjects. On the right, we show a synthetic "surreal" image obtained after rendering the SMPL model using the 3D pose from the real image on the left. Note that for more realism, the surreal image is rendered at the exact same 3D location in the motion capture room, using the same camera and background as in the real image.

Table 2 Comparison with state-of-the-art methods on Human3.6M following protocol P1 that measures an aligned 3D pose distance.

\begin{tabular}{c|ccc}
\hline Method & $\begin{array}{c}\text { 2D source } \\
\text { size }\end{array}$ & $\begin{array}{c}\text { 3D source } \\
\text { size }\end{array}$ & $\begin{array}{c}\text { Error } \\
(\mathrm{mm})\end{array}$ \\
\hline Bo\&Sminchisescu [5] & 120,000 & 120,000 & 117.9 \\
Kostrikov\&Gall [26] & 120,000 & 120,000 & 115.7 \\
Iqbal et al. [67] & 300,000 & 380,000 & 108.3 \\
Ours & 207,000 & 190,000 & $\mathbf{8 8 . 1}$ \\
\hline
\end{tabular}

c) the synthetic and real images together. We observe that the classifier has similar performance when trained on synthetic or real images, which means that our image-based rendering engine synthesizes useful data. Furthermore, we can see that the classifier performs much better when trained on synthetic and real images together. This means that our data is different from the original data and allows the classifier to learn better features. Note that we retrain AlexNet from scratch. We found that it performed better than just fine-tuning a model pre-trained on Imagenet (3D error of $88.1 \mathrm{~mm}$ vs $98.3 \mathrm{~mm}$ with fine-tuning).

In Table 2, we compare our results to three state-of-the-art approaches. Our best classifier, trained with a combination of synthetic and real data, outperforms these methods in terms of $3 \mathrm{D}$ pose estimation by a margin. Note that even though we compute $3 \mathrm{D}$ pose error after $3 \mathrm{D}$ alignment, our method initially estimates absolute pose (with orientation w.r.t. the camera). That is not the case of Bo et al. [5] for instance, who estimate a relative pose and do not provide 3D orientation.

Comparison with classical synthetic images. We make additional experiments to further understand how useful our data is with respect to more classical synthetic data, i.e. obtained by rendering a human $3 \mathrm{D}$ model as in $[8,61]$. To do so, we consider the same 190,000 poses from the previous experiments and render the SMPL 3D human mesh model [30] in these exact same poses using the body parameters and texture maps from [61]. To disambiguate the type of synthetic data, we refer to these new rendered images as "surreal" images, as named in [61] and refer to our data as "mosaic" images. Note that for more realism and to allow for a better comparison, we place the $3 \mathrm{D}$ model in the exact same location within the Human3.6M capture room and use the backgrounds and camera parameters of the corresponding views to render the scenes. An example of the resulting surreal images is visualized in Figure 6 where we also show the corresponding original real image as well as our mosaic image obtained for the exact same pose. When the 2D annotations are accurate and consistent, as it is the case with the Human3.6M dataset, our algorithm produces very plausible images that are locally photorealistic and kinematically correct without significant artefacts at the boundaries between the image patches. Note that for this experiment, we use the poses and images from subjects S1, S5, S6, S7 and S8 to generate our synthetic sets, i.e. removing $\mathrm{S} 9$ from the training set. This allows us to also evaluate on a second protocol (P2) employed in [28,57,71] where only these 5 subjects are used for training.

We then performed quantitative evaluation by training the same classifier on different combinations of the 3 types of data (real, mosaic and surreal). When combining 2 or 3 types of data, we alternate the batches of each data type considering the exact same poses in the different batches. In practice, we consider batches of 256 images and train for $80 \mathrm{k}$ iterations, i.e. 110,55 and 37 epochs for respectively 1, 2 or 3 types of data in the training set. We evaluate on the same subset of frames from subject S11 that was used in the previous experiments (protocol P1). The numerical results are given in Figure 7 where we report classification rate, absolute 3D pose error and $3 \mathrm{D}$ pose error after alignment. We can observe that the model trained on real data (green plot) performs significantly worse than the model trained on our synthetic mosaics (blue) both in terms of classification and 3D pose errors. With even less real data available, subject S9 being removed from the training set for this experiment, our data augmentation 

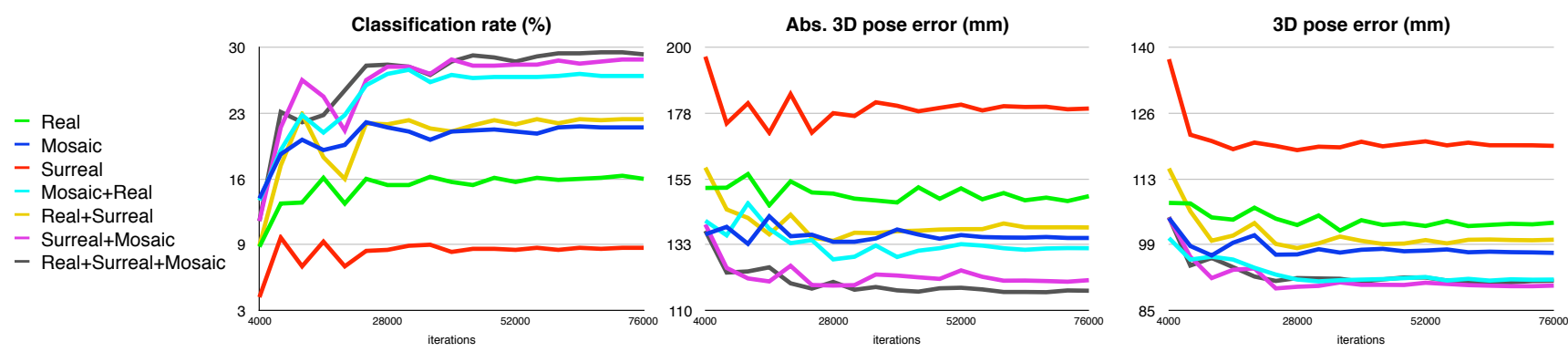

Fig. 7 Human pose estimation results on Human3.6M when training the classifier with different combinations of real, surreal and mosaic data. We report classification rate, absolute 3D pose estimation error and 3D pose error after rigid alignment. We show the performance with respect to the number of training iterations. The three sets of training images all correspond to the same 190,000 3D poses and are labelled with the same class labels.

\begin{tabular}{|c|c|c|c|c|c|c|c|c|c|c|}
\hline Method & $\mathrm{Im}$ & Directions & Discussion & Eat & Greet & Phone & Pose & Purchase & Sit & SitDown \\
\hline Tekin et al.[57] & & 102.4 & 147.7 & 88.8 & 125.3 & 118.0 & 112.3 & 129.2 & 138.9 & 224.9 \\
\hline Zhou et al.[71] & & 87.4 & 109.3 & 87.0 & 103.2 & 116.2 & 106.9 & 99.8 & 124.5 & 199.2 \\
\hline Du et al.[12] & & 85.1 & 112.7 & 104.9 & 122.1 & 139.1 & 105.9 & 166.2 & 117.5 & 226.9 \\
\hline Li et al.[28] & $\checkmark$ & - & 134.1 & 97.4 & 122.3 & - & - & - & - & - \\
\hline Li et al.[29] & $\checkmark$ & - & 133.5 & 97.6 & 120.4 & - & - & - & - & - \\
\hline Tekin et al.[56] & $\checkmark$ & - & 129.1 & 91.4 & 121.7 & - & - & - & - & - \\
\hline Sanzari et al.[46] & $\checkmark$ & 48.8 & 56.3 & 96.0 & 84.8 & 96.5 & 66.3 & 107.4 & 116.9 & 129.6 \\
\hline Zhou et al.[70] & $\checkmark$ & 91.8 & 102.4 & 97.0 & 98.8 & 113.4 & 90.0 & 93.8 & 132.2 & 159.0 \\
\hline Tome et al.[58] & $\checkmark$ & 65.0 & 73.5 & 76.8 & 86.4 & 86.3 & 68.9 & 74.8 & 110.2 & 173.9 \\
\hline Moreno [32] & $\checkmark$ & 69.5 & 80.2 & 78.2 & 87.0 & 100.8 & 76.0 & 69.7 & 104.7 & 113.9 \\
\hline Rogez et al.[44] & $\checkmark$ & 76.2 & 80.2 & 75.8 & 83.3 & 92.2 & 79.0 & 71.7 & 105.9 & 127.1 \\
\hline Pavlakos et al.[36] & $\checkmark$ & 58.6 & 64.6 & 63.7 & 62.4 & 66.9 & 57.7 & 62.5 & 78.6 & 103.5 \\
\hline Ours (real+mosaic) & $\checkmark$ & 94.5 & 110.4 & 109.3 & 143.9 & 125.9 & 95.5 & 89.8 & 134.2 & 179.2 \\
\hline Ours (real+mosaic+surreal) & $\checkmark$ & 87.7 & 100.7 & 93.6 & 139.6 & 107.9 & 88.1 & 78.9 & 119.0 & 171.9 \\
\hline Method & $\mathrm{Im}$ & Smoke & Photo & Wait & Walk & WalkDog & WalkTogether & & Avg. (All) & Avg. (6) \\
\hline Tekin et al.[57] & & 118.4 & 182.7 & 138.7 & 55.1 & 126.3 & 65.8 & & 125.0 & 121.0 \\
\hline Zhou et al.[71] & & 107.4 & 143.3 & 118.1 & 79.4 & 114.2 & 97.7 & & 113.0 & 106.1 \\
\hline Du et al.[12] & & 120.0 & 135.9 & 117.6 & 99.3 & 137.4 & 106.5 & & 126.5 & 118.7 \\
\hline Li et al.[28] & $\checkmark$ & - & 166.2 & - & 68.5 & 132.5 & - & & - & 121.3 \\
\hline Li et al.[29] & $\checkmark$ & - & 163.3 & - & 73.7 & 135.2 & - & & - & 121.6 \\
\hline Tekin et al.[56] & $\checkmark$ & - & 162.2 & - & 65.7 & 130.5 & - & & - & 116.8 \\
\hline Sanzari et al.[46] & $\checkmark$ & 97.8 & 105.6 & 65.9 & 92.6 & 130.5 & 102.2 & & 93.1 & - \\
\hline Zhou et al.[70] & $\checkmark$ & 106.9 & 125.2 & 94.4 & 79.0 & 126.0 & 99.0 & & 107.3 & - \\
\hline Tome et al.[58] & $\checkmark$ & 85.0 & 110.7 & 85.8 & 71.4 & 86.3 & 73.1 & & 88.4 & - \\
\hline Moreno [32] & $\checkmark$ & 89.7 & 102.7 & 98.5 & 79.2 & 82.4 & 77.2 & & 87.3 & - \\
\hline Rogez et al. [44] & $\checkmark$ & 88.0 & 105.7 & 83.7 & 64.9 & 86.6 & 84.0 & & 87.7 & 83.0 \\
\hline Pavlakos et al.[36] & $\checkmark$ & 65.7 & 70.7 & 61.6 & 56.4 & 69.0 & 59.5 & & 66.9 & - \\
\hline Ours (real+mosaic) & $\checkmark$ & 123.8 & 160.3 & 133.0 & 77.4 & 129.5 & 91.3 & & 121.2 & 119.5 \\
\hline Ours (real+mosaic+surreal) & $\checkmark$ & 107.4 & 155.2 & 130.7 & 71.6 & 114.6 & 83.1 & & 110.6 & 112.6 \\
\hline
\end{tabular}

Table 3 Comparison with state of the art on Human3.6M using protocol P2 (average 3D distance without pose alignment). We show per-class results. Im refers to image-based approaches working at the frame level, i.e., that do not leverage temporal information. Note that Du et al. [12] only evaluate on camera 2.

process proves to be even more useful. We can also clearly see that the model trained on surreal images (red plot) does not generalize well to new images as the humans are visibly different. This domain gap problem disappears when the surreal images are mixed with real images (yellow curve). These observations are in par with the conclusions from other works. A domain adaptation process $[8,65]$ or a fine-tuning stage [19] is often required when training on purely synthetic human data. Recently, de Souza et al. [10] also showed in the context of action recognition that overfitting can be avoided by training their classifier on a combination of real and synthetic data. Such domain adaptation is not required when using our mosaic data. We can observe that the model trained on mosaic (blue) performs similarly to the model trained on surreal+real data (yellow) and that adding the real data to the mosaic images results in a better model (cyan). Finally, we can see that combining surreal and mosaic images results in an even better model (magenta curve) and that adding real data to the mosaics and surreal data marginally improves the performance (black). This indicates that our data and the surreal data are complimentary and their association allows to significantly improve the performance when little training data is available. The surreal images bring diversity in terms of body shapes and textures while our mosaics add photo-realism. We believe that we generate hard examples where the symmetry of the body in terms of shape, color and 


\begin{tabular}{ccccccc}
\hline $\begin{array}{c}\text { 2D } \\
\text { source }\end{array}$ & $\begin{array}{c}\text { 3D } \\
\text { source }\end{array}$ & $\begin{array}{c}\text { Number of } \\
\text { training samples }\end{array}$ & $\begin{array}{c}\text { Human3.6M } \\
\text { Abs Error (mm) }\end{array}$ & $\begin{array}{c}\text { Human3.6M } \\
\text { Align. Error (mm) }\end{array}$ & $\begin{array}{c}\text { Human3.6M } \\
\text { Error (pix) }\end{array}$ & $\begin{array}{c}\text { LSP } \\
\text { Error (pix) }\end{array}$ \\
\hline Human3.6M & Human3.6M & 190,000 & 130.1 & 97.2 & 8.8 & 31.1 \\
MPII+LSP & Human3.6M & 190,000 & 248.9 & 122.1 & 17.3 & 20.7 \\
MPII+LSP & CMU & 190,000 & 320.0 & 150.6 & 19.7 & 22.4 \\
MPII+LSP & CMU & $2.10^{6}$ & 216.5 & 138.0 & 11.2 \\
\hline
\end{tabular}

Table 4 Pose error on LSP and Human3.6M using different 2D and 3D sources for rendering the mosaic images and considering different numbers of training samples, i.e., 3D poses and corresponding rendered images.

clothing has not been imposed. This seems to help learning more discriminative features.

In Table 3, we compare our results to state-of-the-art approaches for the second protocol (P2) where all the frames from subjects $\mathrm{S} 9$ and $\mathrm{S} 11$ are used for testing (and only S1, S5, S6, S7 and S8 are used for training). Our best classifier, trained with a combination of synthetic (mosaic \& surreal) and real data, outperforms recent approaches in terms of 3D pose estimation for single frames, even methods such as Zhou et al. [71] who integrate temporal information. Note that our method estimates absolute pose (including orientation w.r.t. the camera), which is not the case for other methods such as Bo et al. [5], who estimate a relative pose and do not provide $3 \mathrm{D}$ orientation. Only the most recent methods report a better performance $[32,36,44,58]$. They use accurate 2D joint detectors $[32,58]$ or rely on much more complex architecture $[36,44]$ while we employ a simple AlexNet architecture and return a coarse pose estimate.

\subsection{Evaluation on Leeds Sport Dataset (LSP)}

We now train our pose classifier using different combinations of training sources and use them to estimate $3 \mathrm{D}$ poses on images captured in-the-wild, i.e., LSP. Since 3D pose evaluation is not possible on this dataset, we instead compare 2D pose errors expressed in pixels and measure this error on the normalized $220 \times 220$ images following [71]. We compute the average $2 \mathrm{D}$ pose error over the 13 joints on both LSP and Human3.6M (see Table 4).

As expected, we observe that when using a pool of the in-the-wild images to generate the mosaic data, the performance increases on LSP and drops on Human3.6M, showing the importance of realistic images for good performance inthe-wild and the lack of generability of models trained on constrained indoor images. The error slightly increases in both cases when using the same number $(190,000)$ of CMU 3D poses. The same drop was observed by [67] and can be explained by the fact that by CMU data covers a larger portions of the 3D pose space, resulting in a worse fit. The results improve on both test sets when considering more poses and synthetic images (2 millions). The larger drop in Abs 3D error and 2D error compared to aligned 3D error means that a better camera view is estimated when using more synthetic data. In all cases, the performance (in pixel) is lower on LSP than on Human3.6M due to the fact that the poses observed in LSP are more different from the ones in the CMU motion capture data. In Figure 8 , we visualize the 2D pose error on LSP and Human3.6M 1) for different pools of annotated 2D images, 2) varying the number of synthesized training images and 3) considering different number of pose classes $K$. As expected using a bigger set of annotated images improves the performance in-the-wild. Pose error converges both on LSP and Human3.6M when using 1.5 million of images; using more than $K=5000$ classes does not further improve the performance. The lower-bound on the $2 \mathrm{D}$ error, i.e., computed with the closest classes from ground-truth annotations, clearly decreases when augmenting the number $K$ of classes. Smaller clusters and finer pose classes are considered when increasing $K$. However, the performance does not further increase for larger values of $K$. The classes become probably too similar, resulting in ambiguities in the classification. Another reasons for this observation could be the amount of training data available for each class that also decreases when augmenting $K$.

Performance with a deeper architecture. To further improve the performance, we also experiment with fine-tuning a VGG-16 architecture [53] for pose classification. By doing so, the average (normalized) 2D pose error decreases by 2.3 pixels. In Table 5, we compare our results on LSP to the state-of-the-art 2D pose estimation methods. Although our approach is designed to estimate a coarse $3 \mathrm{D}$ pose, its performances is comparable to recent $2 \mathrm{D}$ pose estimation methods [9, 66]. In Figure 9, we present some qualitative results obtained with our method. For each image, we show the 3D pose corresponding to the average pose of the top scoring class, i.e., the highest peak in the distribution. The qualitative results in Figure 9 show that our algorithm correctly estimates the global 3D pose. We also show some failure cases.

Re-ranking. After a visual analysis of the results, we found that failures occur in two cases: 1) when the observed pose does not belong to the motion capture training database, which is a limitation of purely holistic approaches (e.g., there exists no motion capture 3D pose of a diver as in the second example on the last row in Figure 9), or 2) when there is a possible right-left or front-back confusion. We observed that this later case is often correct for subsequent top-scoring poses. For the experiments in Table 5 using a VGG architecture, 

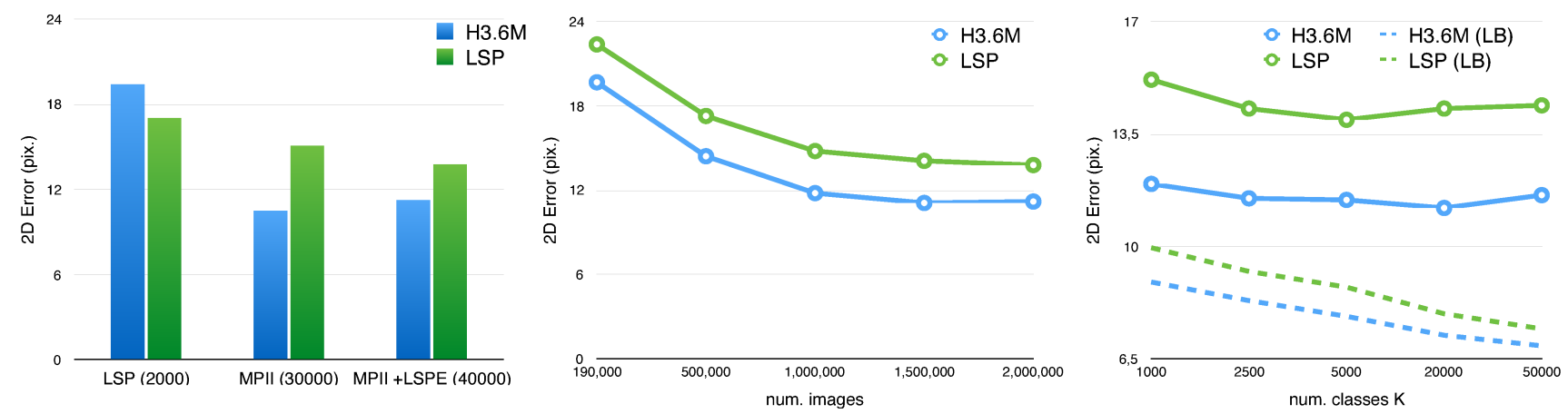

Fig. 8 2D pose error on LSP and Human3.6M using different pools of annotated images to generate 2 millions of mosaic training images (left), varying the number of mosaic training images (center) and considering different number of pose classes $K$ (right). On this last plot, we also report the lower-bound (LB) on the 2D error, i.e., computed with the closest classes from ground-truth annotations.

\begin{tabular}{c|cccccccc}
\hline Method & Feet & Knees & Hips & Hands & Elbows & Shoulder & Head & All \\
\hline Wei et al. [63] & 6.6 & 5.3 & 4.8 & 8.6 & 7.0 & 5.2 & 5.3 & $\mathbf{6 . 2}$ \\
Pishchulin et al. [38] & 10.0 & 6.8 & 5.0 & 11.1 & 8.2 & 5.7 & 5.9 & 7.6 \\
Chen \& Yuille [9] & 15.7 & 11.5 & 8.1 & 15.6 & 12.1 & 8.6 & 6.8 & 11.5 \\
Yang et al. [66] & 15.5 & 11.5 & 8.0 & 14.7 & 12.2 & 8.9 & 7.4 & 11.5 \\
\hline Ours (AlexNet) & 19.1 & 13 & 4.9 & 21.4 & 16.6 & 10.5 & 10.3 & 13.8 \\
Ours (VGG) & 16.2 & 10.6 & 4.1 & 17.7 & 13.0 & 8.4 & 9.8 & 11.5 \\
\hline
\end{tabular}

Table 5 State-of-the-art results on LSP. The 2D pose error in pixels is computed on the normalized $220 \times 220$ images.

the classification rate ${ }^{2}$ on LSP is only $21.4 \%$, meaning that the classes are very similar and very hard to disambiguate. However, this classification rate reaches $48.5 \%$ when considering the best of the 5 top scoring classes, re-ranked using ground truth, as depicted in Fig. 10. It even reaches $100 \%$ when re-ranking the 1000 top scoring classes. i.e., $20 \%$ of the $K=5000$ classes. The $2 \mathrm{D}$ error lower bound $(\approx 8.7$ pixels $)$ is reached when re-ranking the first 100 top scoring classes, only $2 \%$ of the $K=5000$ classes. This highlights a property of our approach that can keep multiple pose hypotheses which could be re-scored adequately, for instance, using temporal information in videos.

\subsection{Discussion}

We now analyse the limitations of the proposed method and discuss future research.

Limitations of the method. In Fig. 11, we show more visual examples of generated images with our approach before and after blending. To better compare the images with/without blending, some close-ups are provided. In general, our imagebased synthesis engine works well when poses and camera views are similar in the query pose and the annotated images. For instance, if the annotated images only include people observed from the front, our engine will not produce acceptable images from side or top views. In the same way, if the

\footnotetext{
2 ground truth classes being obtained by assigning the ground truth $2 \mathrm{D}$ pose to the closest cluster.
}

2D source only contains standing persons, the engine will not be able to synthesise an acceptable image of a sitting pose. If viewpoint and pose are similar in query and annotated images, several factors can influence the quality of the synthesised images. We found three main reasons for failure and show an example of each case in Fig. 12:1) the similarity in person's morphology and clothing in the selected images, in the example given in Fig. 12a, stitching patches of persons wearing trousers or shorts leads to poor result. 2) the 3D depth ambiguities, this inherent to the fact that matching is performed in $2 \mathrm{D}$ and several $3 \mathrm{D}$ poses can correspond to the same 2D pose (see Fig. 12b). 3) the quality of the 2D annotations. While the "perfect" 2D poses from Human3.6M led to very plausible images, this is not always the case for manual annotations of real images. If the $2 \mathrm{D}$ annotations are not consistent or inaccurate, as it often happens with body keypoints such as hips or shoulders, this can results in a synthetic image of poor quality as depicted in Fig. 12c.

Pose and views. A limitation of the proposed approach is that it does not learn the statistics of human poses in real images nor the typical views that can be found in real images. These are two presumably important cues. Ideally, one would want to synthesise useful training images that better match the test conditions. This could be achieved by sampling from a prior distribution of poses and camera viewpoints instead of randomly selecting them as done in this paper.

Image mosaicing. Our mosaic images do not look realistic. While it seems to be more important for the problem at hand to be locally photorealistic as opposed to globally coherent, 

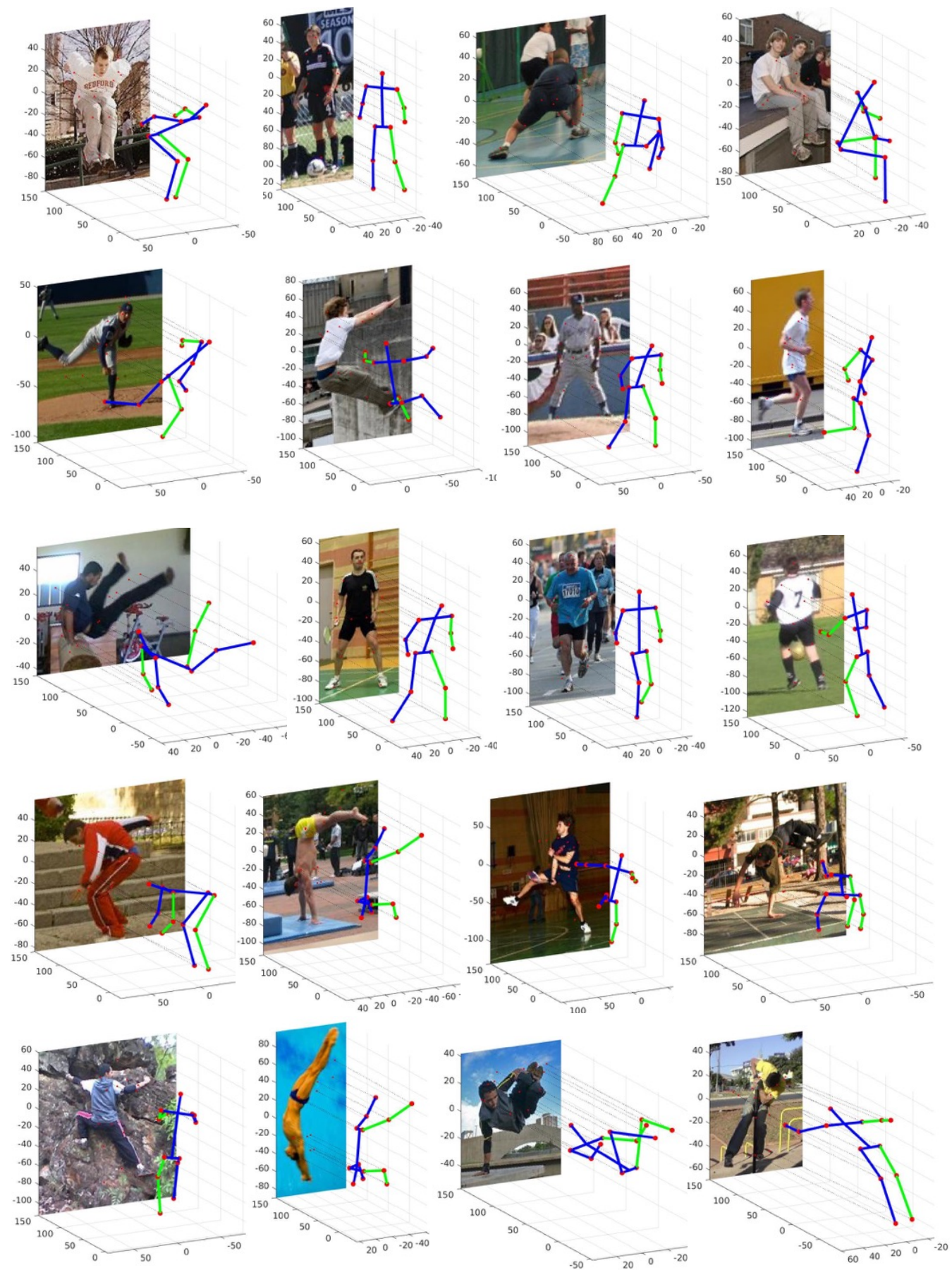

Fig. 9 Qualitative results on LSP. We show for each image, the 3D pose corresponding to the top scoring class, i.e. the highest peak in the distribution. We show correct 3D pose estimations (top 3 rows), imprecise pose estimation due to coarse discretization (fourth row) and typical failure cases (bottom row), corresponding to unseen poses or right-left and front-back confusions.

this is only true for the $3 \mathrm{D}$ pose estimation approaches which were tested in this paper, i.e., deep pipeline with regression or classification objective on images centered on the human. The proposed data might not be appropriate to train more advanced approaches to $3 \mathrm{D}$ pose estimation based on a more global reasoning such as [44] who jointly detect the humans in a natural image and estimate their $3 \mathrm{D}$ poses. The proposed optimization ignores image compatibility, which it could take into account in future work. If a big enough $2 \mathrm{D}$ source, i.e., pool of annotated images, was available, one could constrain the matching in a way that the smallest set of images is used for synthesis, resulting in more satisfactory synthetic images. A new cost function could minimize not just the individual joint scores as a "unary" but also some "binary" 

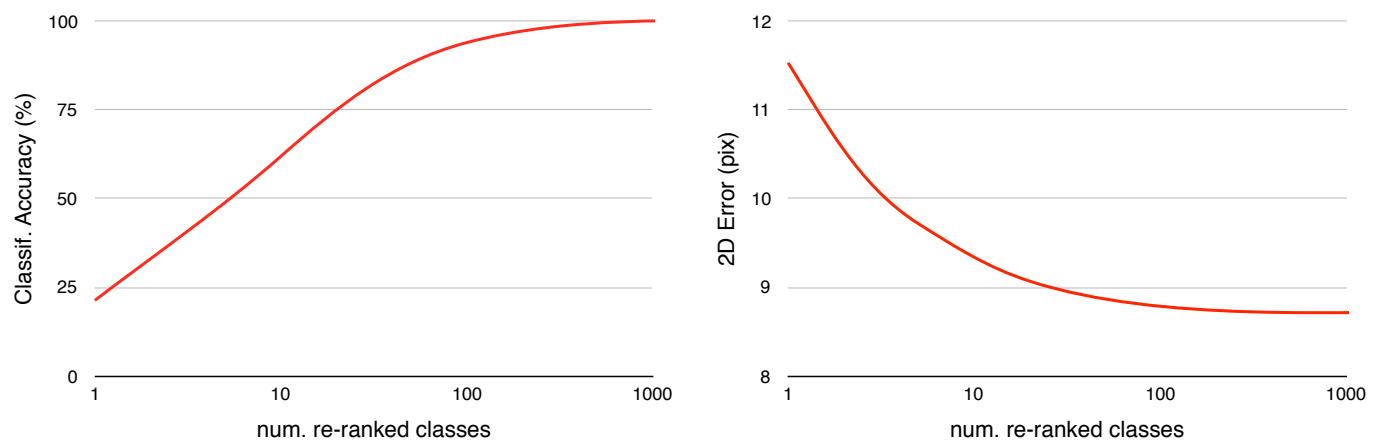

Fig. 10 Classification accuracy and 2D pose error on LSP when re-ranking the top scoring classes. To show the potential improvement of a reranking stage, we report the performance when varying the number of top scoring classes re-ranked using ground truth annotations.
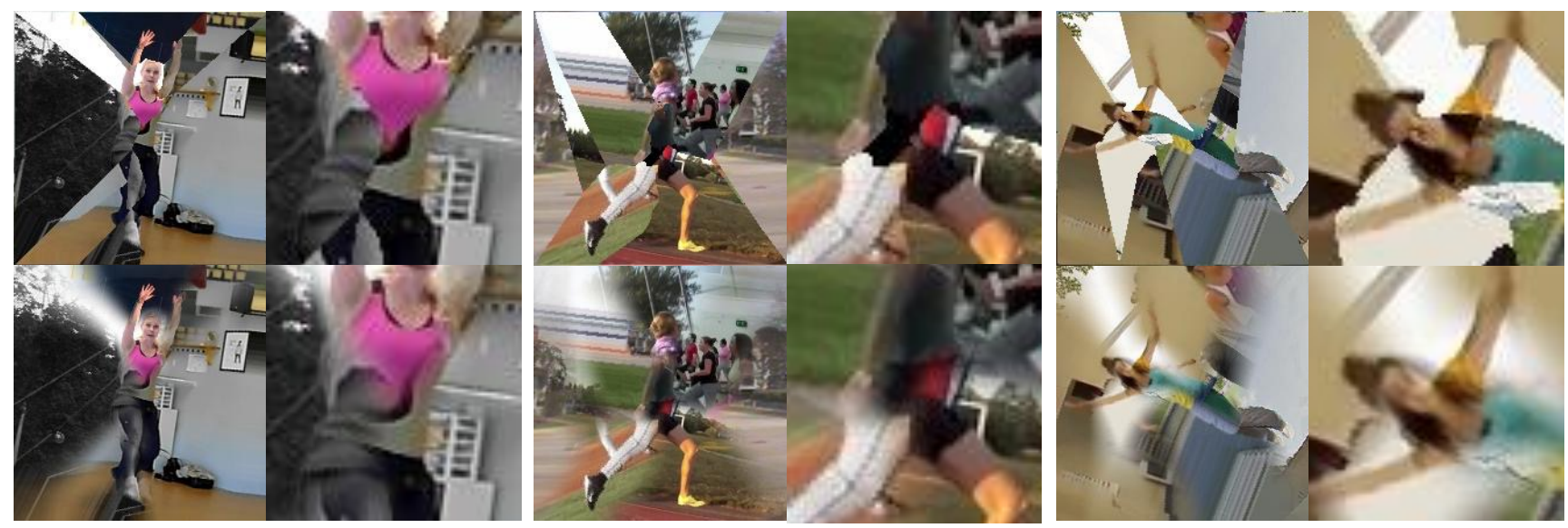

Fig. 11 Examples of synthetic images generated using LSP and MPII datasets and CMU motion capture dataset as 2D and 3D sources respectively. For each case, we show the image before (up) and after (bottom) blending with close-ups.

cost that evaluates the match between pairs of joint matches, and also a prior term that encourages color and geometric consistency/minimal images.

Image blending. In this work, we have proposed to solve the lack of color and geometric consistency with a poseaware blending algorithm that removes the artefacts at the boundaries between image regions while maintaining pose informative edges on the person. In Table 6, we report the performance of the proposed approach without this image blending step and show that this second step is actually necessary. The proposed blending function can seem rather heuristic. Another solution could be a GAN-style [16] image synthesis approach: given images and the probability maps, find a generator to generate images that also defeat a discriminative loss. The resulting images would probably look more compelling and probably respect the overall image structure better (coherent body parts and background geometry). This will be explored in future work. Another intriguing question for future research is whether and to what extent a similar approach could generate synthetic videos with 3D annotations.

\section{Conclusion}

In this paper, we introduce an approach for creating a synthetic training dataset of "in-the-wild" images and their corresponding 3D pose. Our algorithm artificially augments a dataset of real images with new synthetic images showing new poses and, importantly, with 3D pose annotations. We showed that CNNs can be trained on these artificially looking images and still generalize well to real images without requiring any domain adaptation or fine-tuning stage. We train an end-to-end CNN classifier for 3D pose estimation and show that, with our synthetic training images, our method outperforms most published methods in terms of 3D pose estimation in controlled environments while employing a much simpler architecture. We also demonstrated our approach on the challenging task of estimating 3D body pose of humans in natural images (LSP). Finally, our experiments highlight that $3 \mathrm{D}$ pose classification can outperform regression in the large data regime, an interesting and not necessarily intuitive conclusion. In this paper, we have estimated a coarse 3D pose by returning the average pose of the top scoring cluster. In future work, we will investigate how top scoring classes could be re-ranked and also how the pose could be refined. 


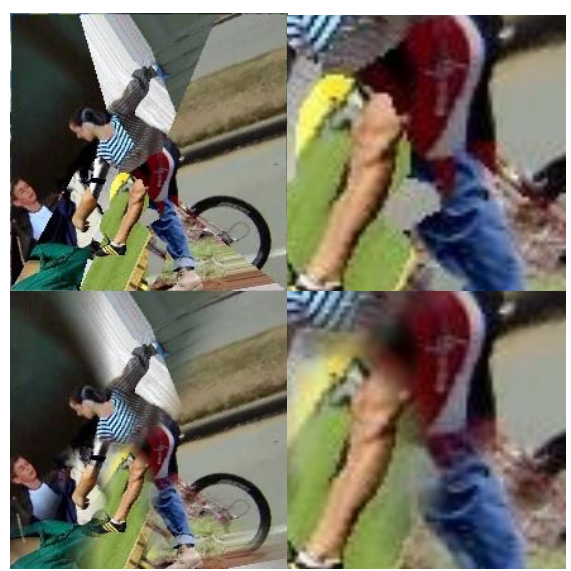

(a)

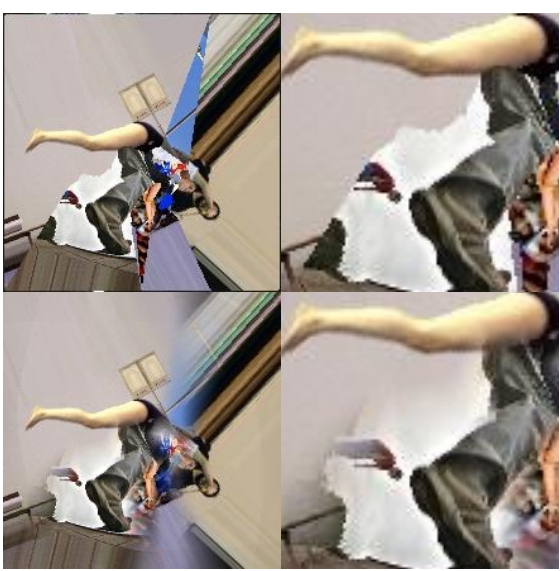

(b)

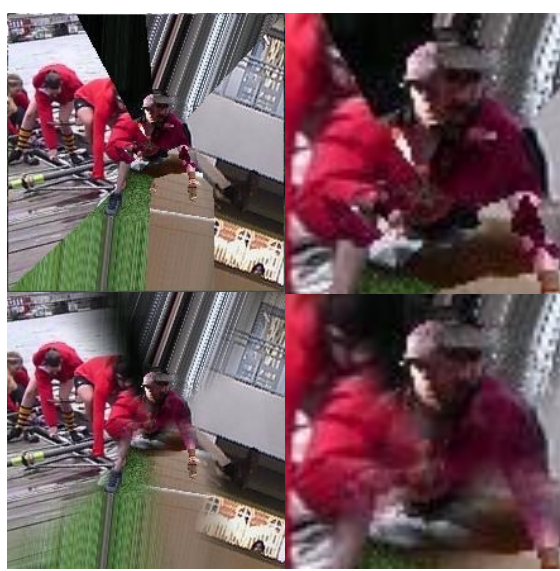

(c)

Fig. 12 Examples of failure cases for synthetic images generated using LSP and MPII datasets and CMU motion capture dataset as 2D and 3D sources respectively. For each case, we show the image before (up) and after (bottom) blending with close-ups. We show failures due to differences in clothing (a), 3D depth ambiguity (b) and inaccurate 2D pose annotations (c).

\begin{tabular}{ccccc}
\hline $\begin{array}{c}\text { Pose-aware } \\
\text { Blending }\end{array}$ & $\begin{array}{c}\text { Human3.6M } \\
\text { Abs Error }(\mathrm{mm})\end{array}$ & $\begin{array}{c}\text { Human3.6M } \\
\text { Error }(\mathrm{mm})\end{array}$ & $\begin{array}{c}\text { Human3.6M } \\
\text { Error (pix) }\end{array}$ & $\begin{array}{c}\text { LSP } \\
\text { Error (pix) }\end{array}$ \\
\hline yes & $\mathbf{3 2 0 . 0}$ & $\mathbf{1 5 0 . 6}$ & $\mathbf{1 9 . 7}$ & $\mathbf{2 2 . 4}$ \\
no & 337.6 & 186.2 & 22.8 & 35.9 \\
\hline
\end{tabular}

Table 6 Pose error on LSP and Human3.6M with and without blending stage. We compare the results obtained by the AlexNet architecture when trained on 190,000 mosaic images synthesized using CMU motion capture 3D poses and MPII+LSP dataset as 3D and 2D sources respectively.

Acknowledgments. This work was supported by the European Commission under FP7 Marie Curie IOF grant (PIOFGA-2012-328288) and partially supported by the ERC advanced grant ALLEGRO and an Amazon Academic Research Award (AARA). We acknowledge the support of NVIDIA with the donation of the GPUs used for this research. We thank Dr Philippe Weinzaepfel for his help. We also thank the anonymous reviewers for their comments and suggestions that helped improve the paper.

\section{References}

1. A. Agarwal and B. Triggs. Recovering 3D human pose from monocular images. PAMI, 28(1):44-58, 2006.

2. I. Akhter and M. Black. Pose-conditioned joint angle limits for 3D human pose reconstruction. In CVPR, 2015.

3. M. Andriluka, L. Pishchulin, P. Gehler, and B. Schiele. 2D human pose estimation: New benchmark and state-of- the-art analysis. In CVPR, 2014.

4. A. Bissacco, M.-H. Yang, and S. Soatto. Detecting humans via their pose. In NIPS, 2006.

5. L. Bo and C. Sminchisescu. Twin Gaussian processes for structured prediction. IJCV , 87(1-2):28-52, 2010.

6. L. Bourdev and J. Malik. Poselets: Body part detectors trained using 3D human pose annotations. In ICCV, 2009.

7. Ching-Hang Chen and Deva Ramanan. 3D human pose estimation $=2 \mathrm{D}$ pose estimation + matching. In CVPR, 2017.

8. W. Chen, H. Wang, Y. Li, H. Su, Z. Wang, C. Tu, D. Lischinski, D. Cohen-Or, and B. Chen. Synthesizing training images for boosting human 3D pose estimation. In 3DV, 2016.

9. X. Chen and A. L. Yuille. Articulated pose estimation by a graphical model with image dependent pairwise relations. In NIPS, 2014.
10. C. R. de Souza, A. Gaidon, Y. Cabon, and A.M. Lopez. Procedural generation of videos to train deep action recognition networks. In CVPR, 2017.

11. A. Dosovitskiy, P. Fischer, E. Ilg, P. Häusser, C. Hazirbas, V. Golkov, P. van der Smagt, D. Cremers, and T. Brox. Flownet: Learning optical flow with convolutional networks. In ICCV, 2015.

12. Y. Du, Y. Wong, Y. Liu, F. Han, Y. Gui, Z. Wang, M. Kankanhalli, and W. Geng. Marker-less 3D human motion capture with monocular image sequence and height-maps. In ECCV, 2016.

13. A. Elhayek, E. Aguiar, A. Jain, J. Tompson, L. Pishchulin, M. Andriluka, C. Bregler, B. Schiele, and C. Theobalt. Efficient convnetbased marker-less motion capture in general scenes with a low number of cameras. In CVPR, 2015.

14. M. Enzweiler and D. M. Gavrila. A mixed generativediscriminative framework for pedestrian classification. In CVPR, 2008.

15. X. Fan, K. Zheng, Y. Zhou, and S. Wang. Pose locality constrained representation for 3D human pose reconstruction. In ECCV, 2014.

16. I.J. Goodfellow, J. Pouget-Abadie, M. Mirza, B. Xu, D. WardeFarley, S. Ozair, A. C. Courville, and Y. Bengio. Generative adversarial nets. In NIPS, 2014.

17. H. Hattori, V. N. Boddeti, K. M. Kitani, and T. Kanade. Learning scene-specific pedestrian detectors without real data. In $C V P R$, 2015.

18. A. Hornung, E. Dekkers, and L. Kobbelt. Character animation from 2D pictures and 3D motion data. ACM Trans. Graph., 26(1), 2007.

19. S. Huang and D. Ramanan. Expecting the unexpected: Training detectors for unusual pedestrians with adversarial imposters. In CVPR, 2017

20. C. Ionescu, D. Papava, V. Olaru, and C. Sminchisescu. Human3.6M: Large scale datasets and predictive methods for 3D human sensing in natural environments. PAMI, 36(7):1325-1339, 2014. 
21. M. Jaderberg, K. Simonyan, A. Vedaldi, and A. Zisserman. Reading text in the wild with convolutional neural networks. IJCV, 116(1):120, 2016.

22. M. Jaderberg, K. Simonyan, A. Zisserman, and K. Kavukcuoglu. Spatial transformer networks. In NIPS, 2015.

23. S. Johnson and M. Everingham. Clustered pose and nonlinear appearance models for human pose estimation. In BMVC, 2010.

24. S. Johnson and M. Everingham. Learning effective human pose estimation from inaccurate annotation. In $C V P R, 2011$.

25. H. Joo, H. Liu, L. Tan, L. Gui, B. Nabbe, I. Matthews, T. Kanade, S. Nobuhara, and Y. Sheikh. Panoptic studio: A massively multiview system for social motion capture. In ICCV, 2015.

26. I. Kostrikov and J. Gall. Depth sweep regression forests for estimating 3D human pose from images. In $B M V C, 2014$.

27. A. Krizhevsky, I. Sutskever, and G. E. Hinton. Imagenet classification with deep convolutional neural networks. In NIPS, 2012.

28. S. Li, W. Zhang, and A. B. Chan. Maximum-margin structured learning with deep networks for 3D human pose estimation. In ICCV, 2015

29. S. Li, W. Zhang, and A. B. Chan. Maximum-margin structured learning with deep networks for 3D human pose estimation. IJCV, 2016.

30. M. Loper, N. Mahmood, J. Romero, G. Pons-Moll, and M. J. Black. SMPL: A skinned multi-person linear model. ACM Trans. Graphics (Proc. SIGGRAPH Asia), 34(6):248:1-248:16, 2015.

31. D. Mehta, H. Rhodin, D. Casas, P. Fua, O. Sotnychenko, W. Xu, and $\mathrm{C}$. Theobalt. Monocular 3D human pose estimation in the wild using improved CNN supervision. In 3D Vision (3DV), 2017.

32. F. Moreno-Noguer. 3D human pose estimation from a single image via distance matrix regression. In $C V P R, 2017$.

33. G. Mori and J. Malik. Recovering 3D human body configurations using shape contexts. PAMI, 28(7):1052-1062, 2006.

34. R. Okada and S. Soatto. Relevant feature selection for human pose estimation and localization in cluttered images. In ECCV, 2008.

35. D. Park and D. Ramanan. Articulated pose estimation with tiny synthetic videos. In CVPR ChaLearn Looking at People Workshop, 2015.

36. G. Pavlakos, X. Zhou, K. G. Derpanis, and K. Daniilidis. Coarseto-fine volumetric prediction for single-image 3D human pose. In CVPR, 2017

37. X. Peng, B. Sun, K. Ali, and K. Saenko. Learning deep object detectors from 3D models. In ICCV, 2015.

38. L. Pishchulin, E. Insafutdinov, S. Tang, B. Andres, M. Andriluka, P. V. Gehler, and B. Schiele. DeepCut: Joint subset partition and labeling for multi person pose estimation. CVPR, 2016.

39. L. Pishchulin, A. Jain, M. Andriluka, T. Thormählen, and B. Schiele. Articulated people detection and pose estimation: Reshaping the future. In $C V P R, 2012$.

40. V. Ramakrishna, T. Kanade, and Y. Sheikh. Reconstructing 3D human pose from 2D image landmarks. In ECCV, 2012.

41. G. Rogez, J. Rihan, C. Orrite, and P. Torr. Fast human pose detection using randomized hierarchical cascades of rejectors. IJCV, 99(1):25-52, 2012.

42. G. Rogez and C. Schmid. MoCap-guided data augmentation for 3D pose estimation in the wild. In NIPS, 2016.

43. G. Rogez, J. Supancic, and D. Ramanan. First-person pose recognition using egocentric workspaces. In CVPR, 2015.

44. G. Rogez, P. Weinzaepfel, and C. Schmid. LCR-Net: LocalizationClassification-Regression for human pose. In CVPR, 2017.

45. J. Romero, H. Kjellstrom, and D. Kragic. Hands in action: Realtime $3 \mathrm{D}$ reconstruction of hands in interaction with objects. In ICRA, 2010.

46. M. Sanzari, V. Ntouskos, and F. Pirri. Bayesian image based 3D pose estimation. In ECCV, 2016.

47. G. Shakhnarovich, P. A. Viola, and T. Darrell. Fast pose estimation with parameter-sensitive hashing. In ICCV, 2003.
48. J. Shotton, A. W. Fitzgibbon, M. Cook, T. Sharp, M. Finocchio, R. Moore, A. Kipman, and A. Blake. Real-time human pose recognition in parts from single depth images. In $C V P R, 2011$.

49. L. Sigal, A. O. Balan, and M. J. Black. Humaneva: Synchronized video and motion capture dataset and baseline algorithm for evaluation of articulated human motion. IJCV, 87(1-2):4-27, 2010.

50. L. Sigal and M. J. Black. Predicting 3D people from 2D pictures. In $A M D O, 2006$

51. E. Simo-Serra, A. Quattoni, C. Torras, and F. Moreno-Noguer. A joint model for $2 \mathrm{D}$ and $3 \mathrm{D}$ pose estimation from a single image. In CVPR, 2013

52. E. Simo-Serra, A. Ramisa, G. Alenyà, C. Torras, and F. MorenoNoguer. Single image 3D human pose estimation from noisy observations. In $C V P R, 2012$.

53. K. Simonyan and A. Zisserman. Very deep convolutional networks for large-scale image recognition. CoRR, abs/1409.1556, 2014.

54. H. Su, C. Ruizhongtai Qi, Y. Li, and L. J. Guibas. Render for CNN: viewpoint estimation in images using $\mathrm{CNNs}$ trained with rendered 3D model views. In ICCV, 2015.

55. Camillo J. Taylor. Reconstruction of articulated objects from point correspondences in a single uncalibrated image. In CVPR, 2000.

56. B. Tekin, I. Katircioglu, M. Salzmann, V. Lepetit, and P. Fua. Structured prediction of $3 \mathrm{D}$ human pose with deep neural networks. In BMVC, 2016.

57. B. Tekin, A. Rozantsev, V. Lepetit, and P. Fua. Direct prediction of 3D body poses from motion compensated sequences. In $C V P R$, 2016.

58. D. Tome, C. Russell, and L. Agapito. Lifting from the deep: Convolutional $3 \mathrm{D}$ pose estimation from a single image. In $C V P R$, 2017.

59. J. J. Tompson, A. Jain, Y. LeCun, and C. Bregler. Joint training of a convolutional network and a graphical model for human pose estimation. In NIPS, 2014.

60. A. Toshev and C. Szegedy. DeepPose: Human pose estimation via deep neural networks. In CVPR, 2014.

61. G. Varol, J. Romero, X. Martin, N. Mahmood, M.J. Black, I. Laptev, and C. Schmid. Learning from synthetic humans. In CVPR, 2017.

62. C. Wang, Y. Wang, Z. Lin, A. L. Yuille, and W. Gao. Robust estimation of 3D human poses from a single image. In CVPR, 2014.

63. S-E Wei, V. Ramakrishna, T. Kanade, and Y. Sheikh. Convolutional pose machines. In $C V P R, 2016$.

64. Z. Wu, S. Song, A. Khosla, F. Yu, L. Zhang, X. Tang, and J. Xiao. 3D shapenets: A deep representation for volumetric shapes. In CVPR, 2015.

65. J. Xu, S. Ramos, D.Vázquez, and A. M. López. Domain adaptation of deformable part-based models. PAMI, 36(12):2367-2380, 2014.

66. W. Yang, W. Ouyang, H. Li, and X. Wang. End-to-end learning of deformable mixture of parts and deep convolutional neural networks for human pose estimation. In CVPR, 2016.

67. H. Yasin, U. Iqbal, B. Krüger, A. Weber, and J. Gall. A dual-source approach for 3D pose estimation from a single image. In $C V P R$, 2016.

68. F. Zhou and F. De la Torre. Spatio-temporal matching for human detection in video. In ECCV, 2014.

69. X. Zhou, Q. Huang, X. Sun, X. Xue, and Y. Wei. Towards 3D human pose estimation in the wild: A weakly-supervised approach. In ICCV, 2017.

70. X. Zhou, X. Sun, W. Zhang, S. Liang, and Y. Wei. Deep kinematic pose regression. In ECCV Workshop on Geometry Meets Deep Learning, 2016.

71. X. Zhou, M. Zhu, S. Leonardos, K. Derpanis, and K. Daniilidis. Sparseness meets deepness: 3D human pose estimation from monocular video. In CVPR, 2016.

72. S. Zuffi and M. J. Black. The stitched puppet: A graphical model of 3D human shape and pose. In $C V P R, 2015$. 